\title{
Prediction of clinical non-response to methotrexate treatment in juvenile idiopathic arthritis
}

\author{
EH Pieter Van Dijkhuizen ${ }^{\text {* }}$, Maja Bulatović ${ }^{1}$, Marloes W Heijstek ${ }^{1}$ Nico M Wulffraat ${ }^{1}$, Saskia MF Pluijm², \\ Robert de Jonge ${ }^{3}$
}

From 18th Pediatric Rheumatology European Society (PReS) Congress

Bruges, Belgium. 14-18 September 2011

\section{Background}

Methotrexate (MTX) is an efficacious drug in juvenile idiopathic arthritis (JIA). If JIA patients are unresponsive to MTX, effective combination treatment with biologicals is required to prevent joint damage.

\section{Aim}

To develop a prediction model to identify MTX nonresponders according to the American College of Rheumatology 70 criteria during the first year of treatment.

\section{Methods}

Data was collected on 183 JIA patients. Clinical variables and single nucleotide polymorphisms (SNPs) in genes involved in the mechanism of action of MTX were determined at baseline. Using multivariate backward logistic regression, these variables were used to construct a prediction model for MTX non-response, whose diagnostic accuracy was evaluated. The model was subsequently validated in a cohort of 104 JIA patients.

\section{Results}

The prediction model included: erythrocyte sedimentation rate and SNPs in genes coding for methionine synthase reductase, multidrug resistance 1 , multidrug resistance protein 1 and proton-coupled folate transporter. The area under the receiver operating characteristics curve (AUROC) was 0.73 (95\%CI: 0.64-0.81). The prediction model was transformed into a total risk score (range 0 to 11 ). At a cut-off score of $\geq 3$, sensitivity was $78 \%$, specificity $49 \%$, positive predictive value was $83 \%$ and negative predictive value $41 \%$. In the validation cohort, the AUROC was 0.65 (95\%CI: 0.540.77).

\section{Conclusion}

The prediction model we developed and validated combines clinical and genetic variables to identify JIA patients not responding to MTX treatment. This model could assist clinicians in making individualized treatment decisions.

\section{Author details}

${ }^{1}$ Department of Pediatric Immunology, University Medical Centre Utrecht, Wilhelmina Children's Hospital, Netherlands. ${ }^{2}$ Department of Public Health, Erasmus University Medical Center Rotterdam, Netherlands. ${ }^{3}$ Department of Clinical Chemistry, Erasmus University Medical Center Rotterdam,

Netherlands.

Published: 14 September 2011

* Correspondence: e.h.p.dijkhuizen@umcutrecht.nl

${ }^{1}$ Department of Pediatric Immunology, University Medical Centre Utrecht, Wilhelmina Children's Hospital, Netherlands

Full list of author information is available at the end of the article 